Submitted to the Annals of Applied Statistics

arXiv: arXiv: 0000.0000

\title{
AN EMPIRICAL BAYES TESTING PROCEDURE FOR DETECTING VARIANTS IN ANALYSIS OF NEXT GENERATION SEQUENCING DATA
}

\author{
By Zhigen ZhAO ${ }^{\dagger}$, WeI WANG ${ }^{\ddagger}$ AND ZHI WeI ${ }^{\ddagger *}$ \\ Temple University ${ }^{\dagger}$ and New Jersey Institute of Technology $y^{\ddagger}$
}

SUPPLEMENTARY MATERIAL

Proof of Theorem 2.1. Firstly,

$$
E \frac{X_{i}}{K_{i}}=\frac{\epsilon}{3}+E \frac{n_{i}}{N}\left(1-\frac{4 \epsilon}{3}\right)=\frac{\epsilon}{3}+\left(1-\frac{4 \epsilon}{3}\right) \pi_{1} \frac{a}{2} .
$$

Therefore, $E m_{1}=\left(1-\frac{4 \epsilon}{3}\right) \pi_{1} \frac{a}{2}$. We then calculate the second moment of $X_{i}$. Since

$$
X_{i} \mid n_{i} \sim b\left(K_{i}, \frac{\epsilon}{3}+\frac{n_{i}}{N}\left(1-\frac{4 \epsilon}{3}\right)\right) .
$$

Then

$$
\begin{aligned}
& E X_{i}^{2} \mid n_{i} \\
= & K_{i}\left(\frac{\epsilon}{3}+\frac{n_{i}}{N}\left(1-\frac{4 \epsilon}{3}\right)\right)\left(1-\frac{\epsilon}{3}-\frac{n_{i}}{N}\left(1-\frac{4 \epsilon}{3}\right)\right)+K_{i}^{2}\left(\frac{\epsilon}{3}+\left(1-\frac{4 \epsilon}{3}\right) \frac{n_{i}}{N}\right)^{2} \\
= & K_{i} \frac{\epsilon}{3}\left(1-\frac{\epsilon}{3}\right)+K_{i}^{2} \frac{\epsilon^{2}}{9}+K_{i}\left(1-\frac{2 \epsilon}{3}\right)\left(1-\frac{4 \epsilon}{3}\right) \frac{n_{i}}{N}+K_{i}^{2} \frac{2 \epsilon}{3}\left(1-\frac{4 \epsilon}{3}\right) \frac{n_{i}}{N} \\
+ & \left(K_{i}^{2}-K_{i}\right)\left(1-\frac{4 \epsilon}{3}\right)^{2} \frac{n_{i}^{2}}{N^{2}} .
\end{aligned}
$$

Further, $E \frac{n_{i}}{N}=\pi_{1} \frac{a^{2}}{2}=E \frac{m_{1}}{\left(1-\frac{4 \epsilon}{3}\right)}$, and

$$
E \frac{n_{i}^{2}}{N^{2}}=\frac{N-1}{N} \pi_{1} \frac{a^{2}}{3}+\frac{1}{N} \pi_{1} \frac{a}{2} .
$$

Consequently,

$$
E m_{2}=E \frac{n_{i}^{2}}{N^{2}}
$$

which completes the proof.

Proof of 2.2.

\footnotetext{
${ }^{*}$ Corresponding Author (E-mail: zhiwei04@gmail.com)
} 
By the definition of $m_{1}$ and $m_{2}$, it is clearly seen that

$$
\operatorname{Var}\left(m_{1}\right)=\frac{\sum_{i} \frac{\operatorname{Var}\left(X_{i}\right)}{K_{i}^{2}}}{p^{2}}=O\left(\frac{1}{p}\right),
$$

and

$$
\operatorname{Var}\left(m_{2}\right)=\frac{1}{p^{2}} \sum_{i} \frac{\operatorname{Var}\left(X_{i}^{2}\right)}{\left(K_{i}^{2}-K_{i}\right)^{2}\left(1-\frac{4 \epsilon}{3}\right)^{4}}=O\left(\frac{1}{p}\right) .
$$

Consequently,

$$
m_{1} \stackrel{\mathrm{P}}{\rightarrow}\left(1-\frac{4 \epsilon}{3}\right) \pi_{1} \frac{a}{2}, m_{2} \stackrel{\mathrm{P}}{\rightarrow} \frac{N-1}{N} \pi_{1} \frac{a^{2}}{3}+\frac{1}{N} \pi_{1} \frac{a}{2} .
$$

Consequently,

$$
\hat{a} \stackrel{P}{\rightarrow} a, \hat{\pi}_{1} \stackrel{\mathrm{P}}{\rightarrow} \pi_{1} .
$$

\section{Proof of Theorem 2.3.}

The proof is similar to the proof of Theorem 3 and 4 in Sun and Wei (2011).

Define $\hat{Q}(t)=\frac{\sum_{i} I\left(\widehat{f d r_{i}}(\boldsymbol{X})<t\right) f \widehat{d r_{i}(\boldsymbol{X})}}{\sum_{i} I\left(f d r_{i}(\boldsymbol{X})<t\right)}$. The $\alpha$-level BFDR cutoff of this empirical Bayes procedure is denoted as $\hat{c}_{E B}$.

According to Theorem 2.2 , we know that $\hat{\pi}_{1} \stackrel{\mathrm{P}}{\rightarrow} \pi_{0}$, and $\hat{a} \stackrel{\mathrm{P}}{\rightarrow} a$, implying that $f d \widehat{r_{i}(\boldsymbol{X})} \stackrel{\mathrm{P}_{\dot{S}}}{\rightarrow} f d r_{i}(\boldsymbol{X})$ based on the Mann-Walk Theorem (Mann and Wald (1943)). Applying the weak law of large numbers for the triangular arrays, we know that

$$
\hat{Q}(t) \stackrel{\mathrm{P}_{\rightarrow}}{\rightarrow} \frac{\sum_{i} I\left(f d r_{i}(\boldsymbol{X})<t\right) f d r_{i}(\boldsymbol{X})}{\sum_{i} I\left(f d r_{i}(\boldsymbol{X})<t\right)}=B F D R_{O R}\left(\boldsymbol{\delta}^{B}(t)\right) .
$$

Let $c_{O R}$ be the oracle cutoff such that $B F D R_{O R}\left(\boldsymbol{\delta}^{B}\left(c_{O R}\right)\right)=\alpha$. Next, we will prove that $\hat{c}_{E B} \stackrel{P}{\rightarrow} c_{O R}$.

Note that $\hat{Q}(t)$ is a constant in the interval $f d \widehat{r_{(i)}}(\boldsymbol{X}) \leq t<f d r_{(i+1)}(\boldsymbol{X})$. As a result,

$$
\begin{aligned}
& \left.\hat{c}_{E B}=\max _{i=1,2, \cdots, p}\left\{f d \widehat{r_{(i)}}(\boldsymbol{X}): \frac{1}{i} \sum_{j=1}^{i} f \widehat{f d r_{(i)}} \widehat{X}\right) \leq \alpha\right\} \\
= & \max _{i=1,2, \cdots, p}\left\{f d \widehat{r_{(i)}}(\boldsymbol{X}): \hat{Q}\left(f d \widehat{r_{(i)}}(\boldsymbol{X})\right) \leq \alpha\right\} \\
= & \sup \{c \in(0,1): \hat{Q}(c) \leq \alpha\}=\hat{Q}^{-1}(\alpha) .
\end{aligned}
$$


We already know that $\hat{Q}(t) \stackrel{\mathrm{P}_{\hookrightarrow}}{\rightarrow} B F D R_{O R}\left(\boldsymbol{\delta}^{B}(t)\right)$. Therefore, $\hat{c}_{E B} \stackrel{\mathrm{P}_{\hookrightarrow}}{\rightarrow} c_{O R}$ based on the functional delta method. As a result,

$$
\begin{aligned}
& B \widetilde{F D} R=E \frac{\sum_{i=1}^{p} I\left(f d \widehat{\left.r_{i}(\boldsymbol{X})<\hat{c}_{E B}\right) f d r_{i}(\boldsymbol{X})}\right.}{\sum_{i=1}^{p} I\left(f d r_{i}(\boldsymbol{X})<\hat{c}_{E B}\right)} \\
= & E \frac{\sum_{i=1}^{p} I\left(f d r_{i}(\boldsymbol{X})<\hat{c}_{E B}\right) f d r_{i}(\boldsymbol{X})}{\sum_{i=1}^{p} I\left(f d r_{i}(\boldsymbol{X})<\hat{c}_{E B}\right)}+o(1) \\
= & E \frac{\sum_{i=1}^{p} I\left(f d r_{i}(\boldsymbol{X})<c_{O R}\right) f d r_{i}(\boldsymbol{X})}{\sum_{i=1}^{p} I\left(f d r_{i}(\boldsymbol{X})<c_{O R}\right)}+o(1) \\
= & B F D R_{O R}+o(1) .
\end{aligned}
$$

Similarly, one can show that $B \widetilde{F N} R=B F N R_{O R}+o(1)$.

Supplement to "An empirical Bayes testing procedure for detecting variants in analysis of next generation sequencing data" (doi: COMPLETED BY THE TYPESETTER; .pdf). This file contains the technical proof of the theorems.

\section{References.}

MAnn, H. B. and WALD, A. (1943). On stochastic limit and order relationships. The Annals of Mathematical Statistics 14 217-226.

Sun, W. and WeI, Z. (2011). Multiple Testing for Pattern Identification, With Applications to Microarray Time-Course Experiments. Journal of the American Statistical Association 106 73-88.

Zhigen Zhao

Department of Statistics

TEMPle University

346 Speakman Hall

1810 N. 13Th Street

Philadelphia, 19122

E-MAIL: zhaozhg@temple.edu
Wei Wang and Zhi Wei

Department of Computer Science

New Jersey Institute of Technology

GITC 4400, University Heights

NEWARK, NJ 07102

E-MAIL: ww42@njit.edu zhiwei@njit.edu

URL: http://ebvariant.sourceforge.net 